\title{
Ferroelectric Switching Characteristics and Crystal Structure of Nylon 11
}

\author{
Yoshiyuki TaKahashi, Masataka Shimomura, Masayuki Kutani,* \\ and Takeo FuruKawA \\ Department of Chemistry, Faculty of Science, Science University of Tokyo, \\ 1-3 Kagurazaka, Shinjuku-ku, Tokyo 162, Japan \\ * Toyoseiki Seisakusho Co., Ltd., \\ 5-15-4 Takinogawa, Kita-ku, Tokyo 114, Japan
}

(Received July 26, 1996)

\begin{abstract}
The time domain measurement of polarization reversal is made for melt-quenched, uniaxially-drawn and annealed Nylon 11 samples by applying a stepwise electric field. The amount of reversed polarization of an as-drawn sample is almost independent of the field strength above $80 \mathrm{MV} \mathrm{m}^{-1}$ and gives a value of $\mathrm{ca}$. $50 \mathrm{mC} \mathrm{m}^{-2}$. The switching time is proportional to a power of the field strength. Annealing above $100^{\circ} \mathrm{C}$ causes a marked increase in switching time but does not change the amount of reversed polarization. X-ray study shows that annealing results in a change in crystal structure from a pseudohexagonal phase containing distorted conformation into an all-trans triclinic phase. Such a structural change enlarges the potential barrier against the chain rotation to result in the increase in the switching time.
\end{abstract}

KEY WORDS Nylon 11 / Ferroelectricity / Structure / Annealing / Switching / Dynamics /

Poly(vinylidene fluoride) and its copolymers are ferroelectrics. Their crystals are polar and the polarity can be switched by an application of an electric field. They exhibit $D-E$ hysteresis loops. The copolymers show the Curie transition as well. The study on ferroelectricity in polymeric material had been centered on these polymers because they were the only ferroelectric polymers. ${ }^{1}$ However, as results of searches for new ferroelectric polymers, increasing was the number of polymers that had possibility of being ferroelectrics. Odd Nylons were ones of such polymers because they have polar crystal structure.

Electric field-induced change of orientation of dipoles was found by Jacobs and Hicks for Nylon 11.2 The piezoelectric constant showed a hysteresis against a change of poling field. ${ }^{3,4}$ Finally, after detailed research on odd Nylons, Lee and coworkers succeeded in observing $D-E$ hysteresis loops in Nylons 7 and 11 by measuring a current from a sample capacitor subjected to an alternating voltage. ${ }^{5}$ They found ferroelectricity in a sample prepared by a limited condition, i.e., by stretching a melt-quenched film. They also reported that annealing causes changes in the crystal structure and leads to loss of ferroelectricity of odd Nylons. ${ }^{6}$

The $D-E$ hysteresis measurement is a useful method to see whether the material is ferroelectric or not. However, the results obtained at a fixed frequency contain uncertainty because polarization reversal giving rise to a $D-E$ hysteresis loop is essentially a dynamical process. In this paper, we report on our dynamical investigation of polarization reversal in Nylon 11 by means of switching measurements. We discuss the effect of annealing on switching characteristics in connection with structural changes revealed by X-ray measurements.

\section{EXPERIMENTAL}

Pellets of Nylon 11 manufactured by Rilsan Co. were melt-pressed at $210^{\circ} \mathrm{C}$ and quenched in liquid nitrogen. Films obtained were then drawn uniaxially to three times at room temperature. The thickness of resulted films was about $15 \mu \mathrm{m}$. We designate them as-drawn samples. To examine the effect of annealing, as-drawn samples were kept at temperatures from $75^{\circ} \mathrm{C}$ to $175^{\circ} \mathrm{C}$ for an hour. Gold was evaporated onto both surfaces of sample films as electrodes.

The dynamics of polarization reversal was studied by switching measurements in which a step voltage was applied to the sample and the response in charge was measured as a function of time. The electric displacement is obtained as a response in charge per unit area of the electrode. The response consists of contributions from the polarization reversal and the dielectric polarization as well as the DC conduction when the applied field is reverse to the existing polarization in the sample. If the field is in the same direction as, or forward to, the polarization, only the latter two contribute to the electric displacement. Therefore the reverse response subtracted by the forward response gives just the contribution of the polarization reversal. In this paper, the electric displacement $D(t)$ is corrected in this way.

Crystal structure was studied by flat-plate X-ray photography using nickel-filtered $\mathrm{Cu}-K_{\alpha}$ radiation. Three kinds of geometry were adopted: the incident Xray beam parallel to the draw direction (end-mode), parallel to the normal of the film (through-mode) and perpendicular to both the draw direction and the normal of the film (edge-mode).

\section{RESULTS AND DISCUSSION}

Figure 1 shows the results of switching measurements in that the electric displacement of an as-drawn sample at various field strength is plotted against the logarithm of time $t$. Sigmoidal curves characteristic to ferroelectric polarization reversal are clearly seen. The value of the electric displacement finally reached gives double the amount of the reversed polarization $P_{\mathrm{r}}$ which is about $50 \mathrm{mC} \mathrm{m}^{-2}$ being nearly independent of the field strength.

The electric displacement differentiated by the log- 


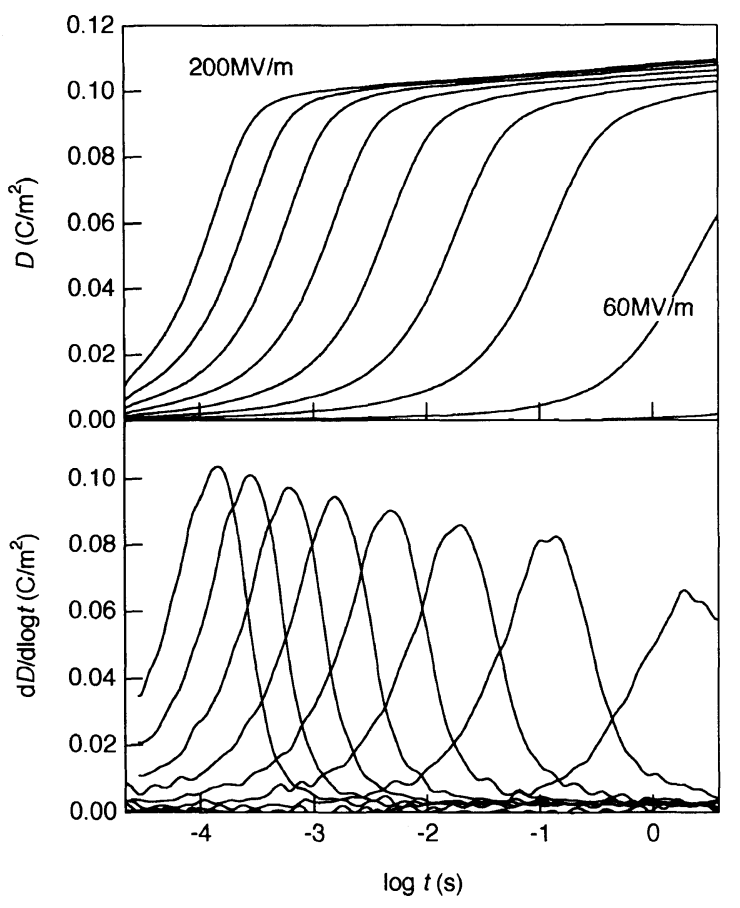

Figure 1. Switching curves of an as-drawn sample at various fields.

arithm of time $\mathrm{d} D / \mathrm{d} \log t$ shows a peak at a time that defines the switching time $\tau_{s}$. As shown in Figure 1, the switching time decreases with increasing the strength of the field. This means that the switching process of Nylon 11 is field-activated as is the case for most ferroelectrics.

Figure 2 shows the switching curves at $200 \mathrm{MV} \mathrm{m}^{-1}$ of samples annealed at various temperatures. It is seen that annealing strongly affects the dynamical characteristics of the switching process: the switching process is slowed and the switching curve becomes broader as the annealing temperature is raised, while the amount of reversed polarization remains unchanged until the annealing temperature reaches $175^{\circ} \mathrm{C}$. Figure 3 shows the annealing temperature dependence of the switching time at $200 \mathrm{MV} \mathrm{m}^{-1}$. The switching time rapidly increases with annealing temperature above $100^{\circ} \mathrm{C}$.

The field strength dependence of the switching times of these samples is summarized in Figure 4 in which the logarithm of the switching time is plotted against the logarithm of the field strength. The linear relationship means that the switching time $\tau_{\mathrm{s}}$ is proportional to a power of the field $E$ as

$$
\tau_{\mathrm{s}}=A E^{-m}
$$

where $m$ is an exponent and $A$ is a constant. In Figure $5, A$ and $m$ are plotted as a function of annealing temperature. Both of them increase with annealing temperature above $100^{\circ} \mathrm{C}$ in accord with the change in the switching time. As shown in Figure 2, annealing also causes a marked broadening of the derivative of switching curve. This indicates that the polarization reversal proceeds over a broader range of time in the annealed sample.

Lee et al. have shown that the remanent polarization obtained from $D-E$ hysteresis measurement is decreased by annealing. ${ }^{6}$ Their results seem to suggest that ferroelectricity is lost by annealing at higher temperatures. This is shown to be not the case by our switching mea-

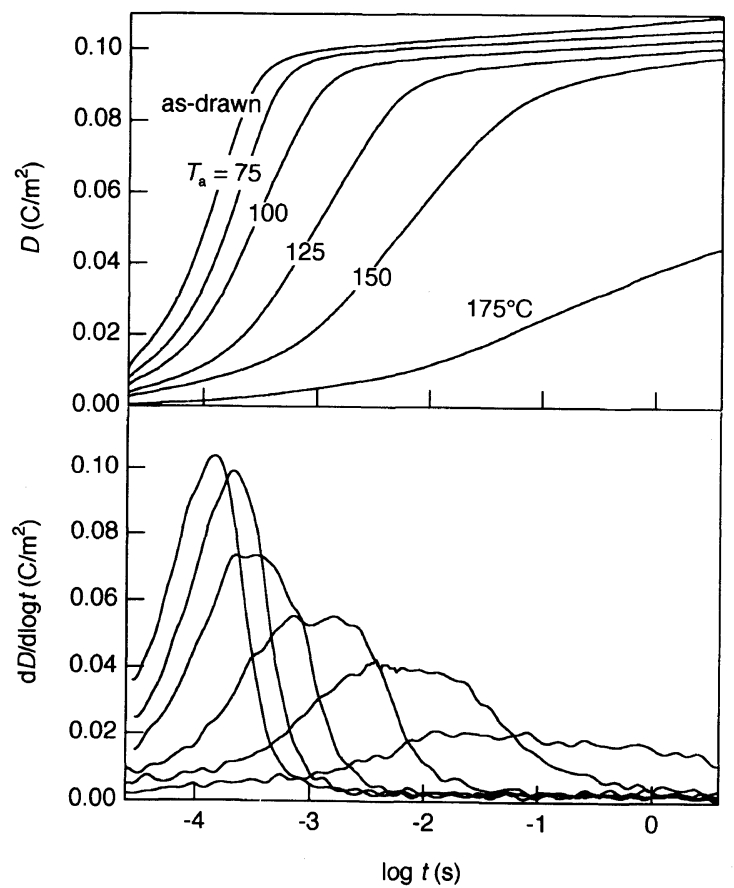

Figure 2. Switching curves of samples annealed at various temperatures. The field is $200 \mathrm{MV} \mathrm{m}^{-1}$.

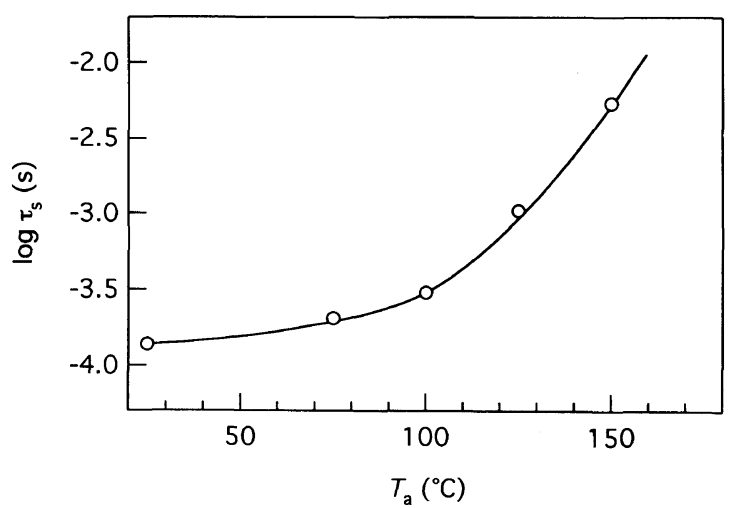

Figure 3. Annealing temperature dependence of the switching time.

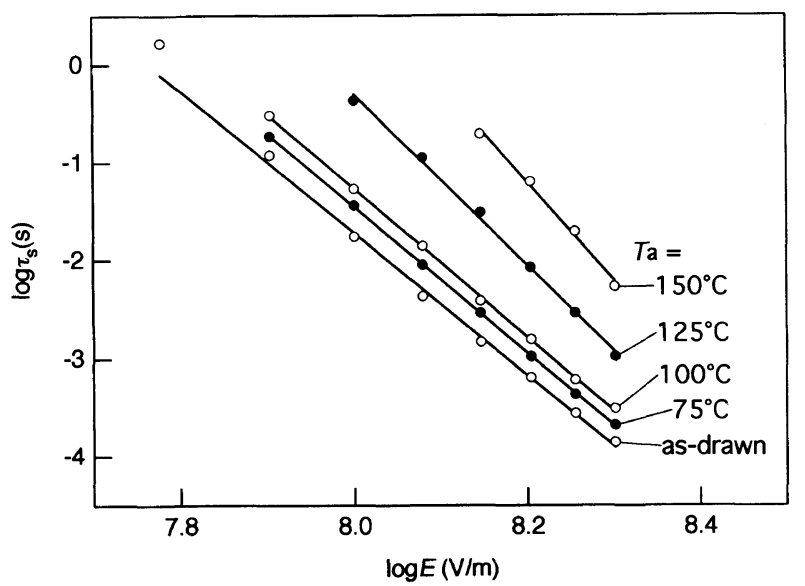

Figure 4. Electric field dependence of the switching time of samples annealed at various temperatures.

surements. Since annealing causes slowing as well as broadening of switching process, annealed sample cannot catch up the change of electric field to result in a diminished $D-E$ hysteresis loop at a fixed frequency. 
Recently Ikeda et al. have reported the switching curves in Nylon $11 .^{7}$ However, their results are different from ours: the reversed polarization in their report depends

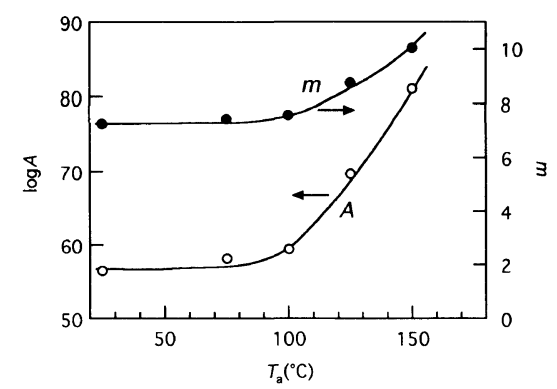

Figure 5. Annealing temperature dependence of constants $A$ and $m$.
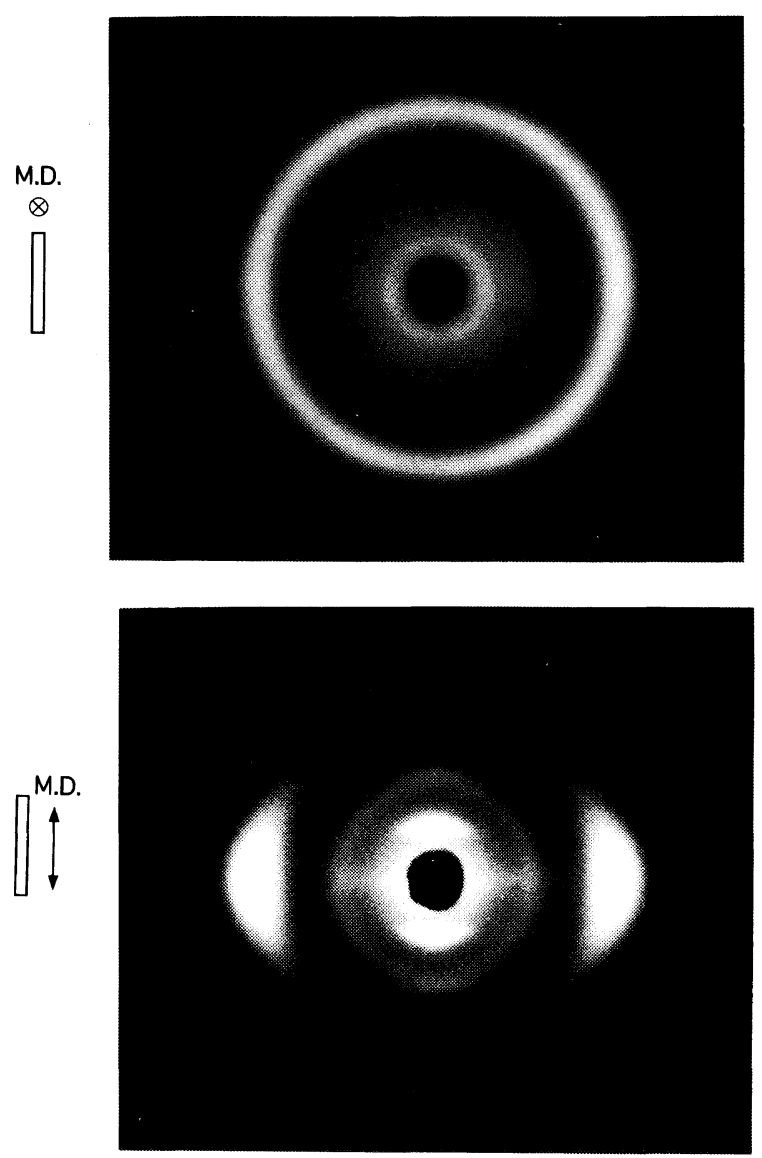

(b)

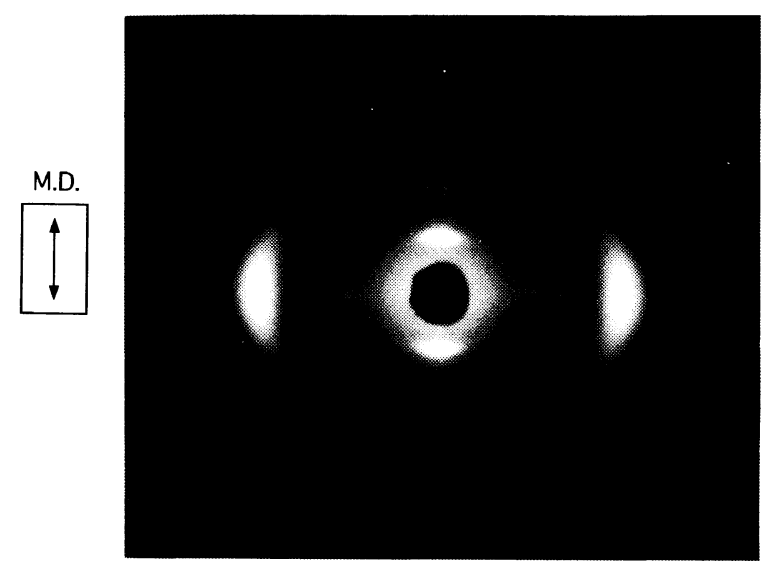

Figure 6. X-ray photographs of an as-drawn sample. (a) end-mode; (b) edge-mode; (c) through-mode. M.D.'s indicate the machine direction and rectangles show the specimen geometry. on the field strength while it does not in our work. In addition, they reported that the switching time is not affected by annealing contrarily to our results. These difference may be because the ferroelectric properties depend strongly on the condition of preparing the specimen. It is to be noted that our results are extremely reproducible for the samples prepared in the condition adopted in this work.

The structural change on annealing was examined by $\mathrm{X}$-ray photography. Figure 6 shows the X-ray photographs of an as-drawn sample. Though the diffractions are rather diffusive, a single diffraction ring in the endmode photograph indicates that the crystal system is pseudohexagonal. Annealing causes dramatic changes in $\mathrm{X}$-ray diffraction pattern as demonstrated in Figure 7 which is a set of photographs of a sample annealed at $175^{\circ} \mathrm{C}$. The end-mode photograph shows several arcs with different spacings. The spacing of a plane, which is parallel to the film surface corresponding to the diffraction $\mathrm{A}$ in Figure 7(a), is decreased by annealing although that corresponding to $B$ is unchanged. The edge-mode photograph shows a split of meridional diffraction: (001) plane is not orthogonal to the chain axis. Thus the crystal system of an annealed Nylon 11 is triclinic.

Figure 7 also shows that the crystals are doubly oriented, although double orientation is not obvious in the as-drawn sample because of pseudohexagonal symmetry (Figure 6(a)). It is plausible to assume that the change from uniaxial to double orientation does not occur on annealing. Therefore the orientation in the as-drawn sample can be revealed by annealing. Poling of an as-drawn sample yields an end-mode photograph as shown in Figure 8(a) which hardly differs from Figure 6(a). We then annealed the poled sample at $175^{\circ} \mathrm{C}$ to obtain Figure $8(\mathrm{~b})$. Diffractions of two different spacings are observed as indicated by $\mathrm{C}$ and $\mathrm{D}$. The lattice plane which gives the diffraction $\mathrm{D}$ has smaller spacing than that corresponding to $\mathrm{C}$. This is consistent with an annealing induced change in crystal system from pseudohexagonal to triclinic as observed with respect to an unpoled sample, although annealing decreases the spacing of the plane which makes an angle of $c a .60$ degrees to the film surface. The diffractions indicated by $\mathrm{A}$ and by B in Figure 7(a) are thus transferred to D and $\mathrm{C}$ in Figure 8(b) respectively. This means that crystals are rotated by 60 degrees about the chain axis.

A dipole in Nylon 11 molecule is located at the amide group which is hydrogen-bonded to one another to make up a hydrogen-bonded sheet. Because dipoles are rotated by poling, the hydrogen-bonded sheet is also rotated toward the field direction. Owing to the pseudohexagonal symmetry of packing of chains in the as-drawn crystal, the orientation of the crystal can be rotated by an angle of a multiple of 60 degrees as a result of rotation of constituent chains with no translational displacement. As for a chain the dipoles of which are parallel to the film surface, rotation of 60 degrees is most probable. This is the case for diffraction A in Figure 7(a) and D in Figure 8(b). Thus it is suggested that the dipoles, and therefore the hydrogen-bonded sheets, are oriented in parallel to the film surface before poling and are rotated by an angle of 60 degrees by poling. 

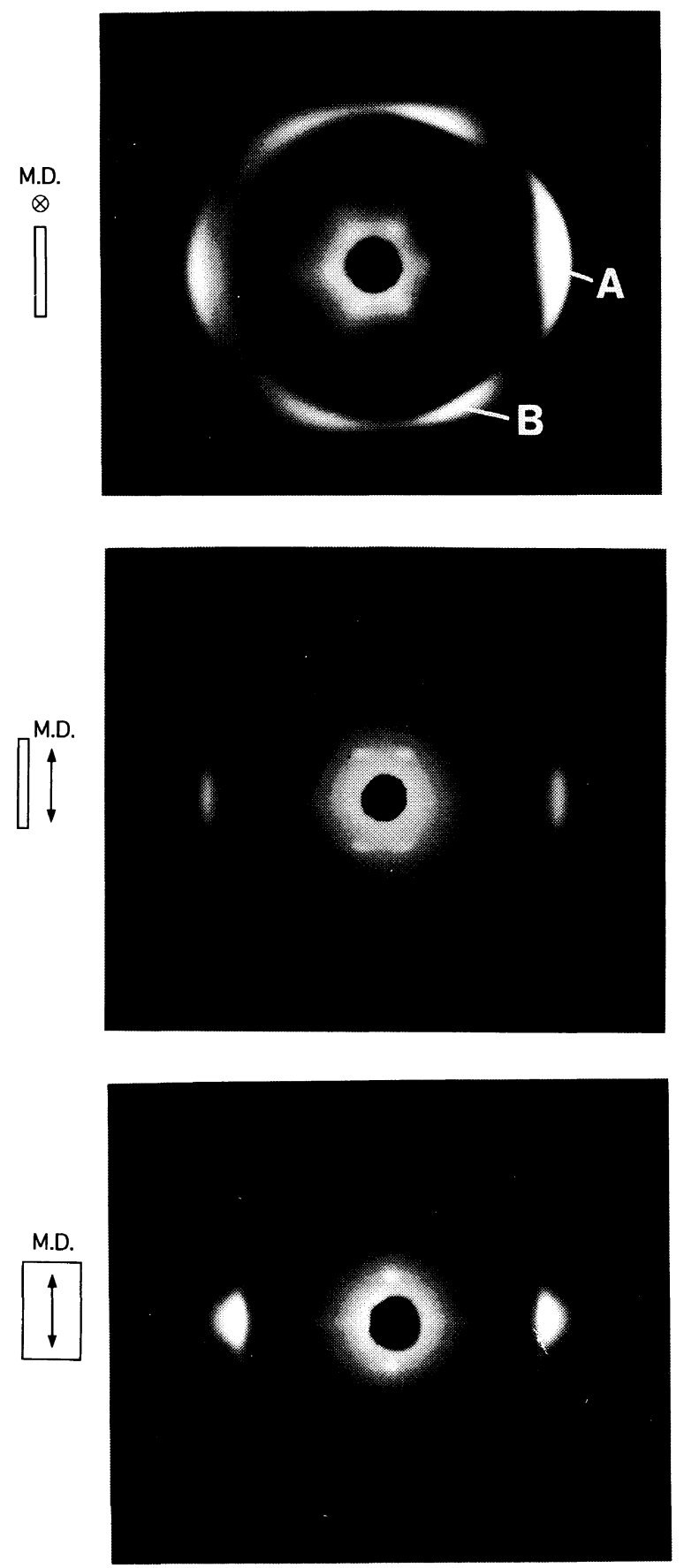

Figure 7. X-ray photographs of a sample annealed at $175^{\circ} \mathrm{C}$ (a) end-mode; (b) edge-mode; (c) through-mode.

The change in diffraction pattern was also reported by Scheinbeim et al. ${ }^{8,9}$ They have shown that both the rotation by 60 degrees and by 90 degrees are possible depending on the humidity. ${ }^{9}$ Our poling process is performed in the air without controlling the humidity. The inconsistency between their results and ours is not clear.

The X-ray photograph of through-mode (Figure 6(c)) shows that 001 reflection is on the meridian for the as-drawn sample. So we can take a rectangular lattice in the hydrogen-bonded sheet. The $c$-axis is parallel to the chain direction and the $a$-axis is perpendicular to it. To make up a rectangular lattice, the unit cell must have at least two chains antiparallel to each other. In this way, we assign an index of 200 to $B$ and $C$ and 010 to $A$ and D.

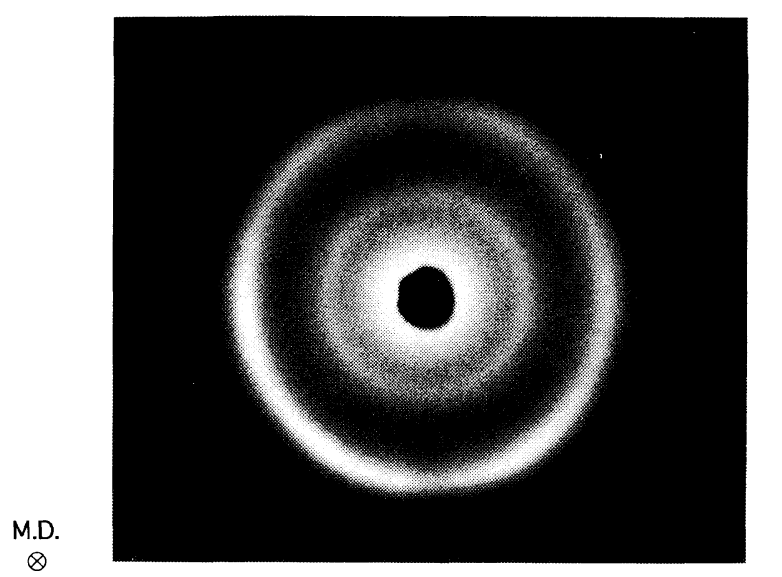

(a)

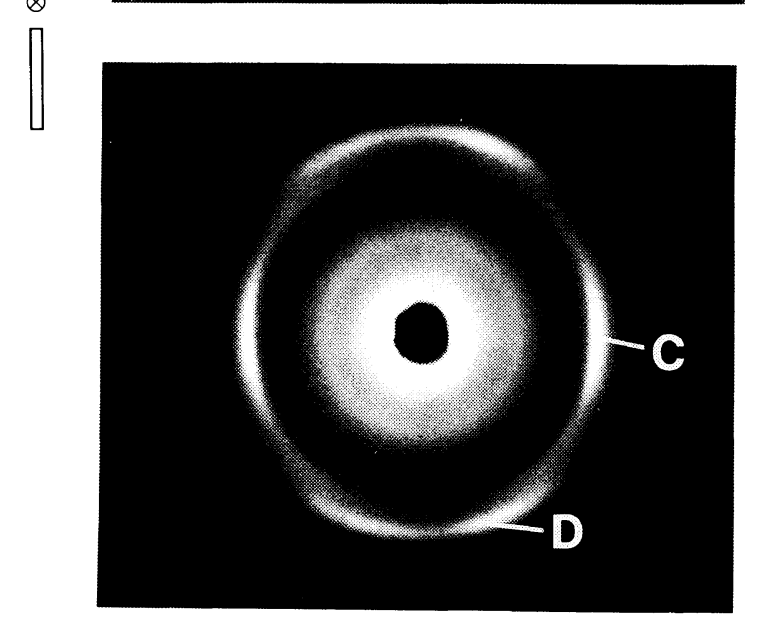

(b)

Figure 8. X-ray photographs of end-mode of samples (a) as-drawn and poled and (b) as-drawn, poled and annealed at $175^{\circ} \mathrm{C}$.

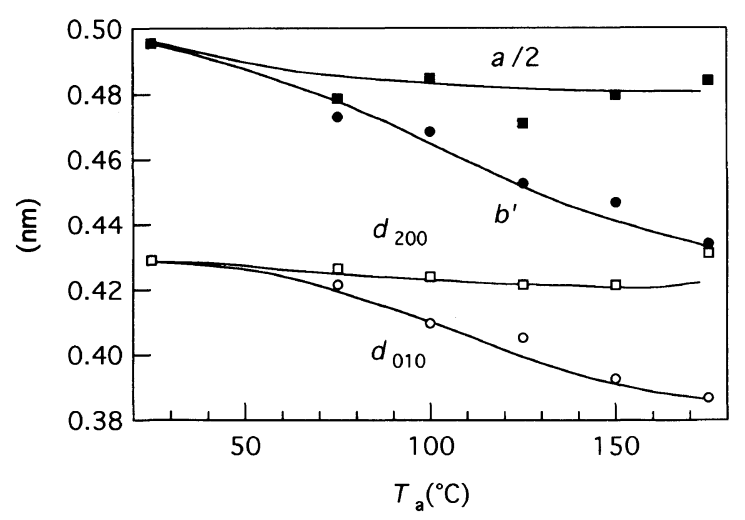

Figure 9. Annealing temperature dependence of spacing $d_{200}$ and $d_{010} . a / 2$ and $b^{\prime}$ are also shown.

The annealing temperature dependence of $d_{200}$ and $d_{010}$, the spacing of (200) and (010) respectively, is shown in Figure 9. Also shown is that of $a / 2$ and $b^{\prime}$. Here, $d_{010}$ is the spacing between the hydrogen-bonded sheets and $a / 2$ is the distance between chains which are hydrogen-bonded to each other. The change in $d_{010}$ shows that the spacing of hydrogen-bonded sheet decreases with annealing temperature above $100^{\circ} \mathrm{C}$. In contrast to $d_{010}, a / 2$ remains unchanged which suggests that the strength of hydrogen-bonding is hardly affected by annealing.

Figure 10 shows the annealing temperature dependence of the fiber period $c$ as well as that of the spacing $d_{001}$ and $\alpha$, the angle between the $b$ - and $c$-axes. The angle $\alpha$ 
changes off the right angle on annealing which means that the hydrogen-bonded sheet glides along the $c$-axis. The fiber period $c$ calculated from $d_{001}$ and $\alpha$ changes with annealing temperature above $100^{\circ} \mathrm{C}$ in parallel with the change in the spacing $d_{200}$.

By comparing Figure 3 and Figure 5 with Figure 9 and Figure 10, it is clear that the changes in dynamical properties of switching corresponds well to the change in the structure. Since the amount of reversed polarization is unchanged by annealing, the structural change must be such that strongly affects only the dynamical properties. We propose possible structures of

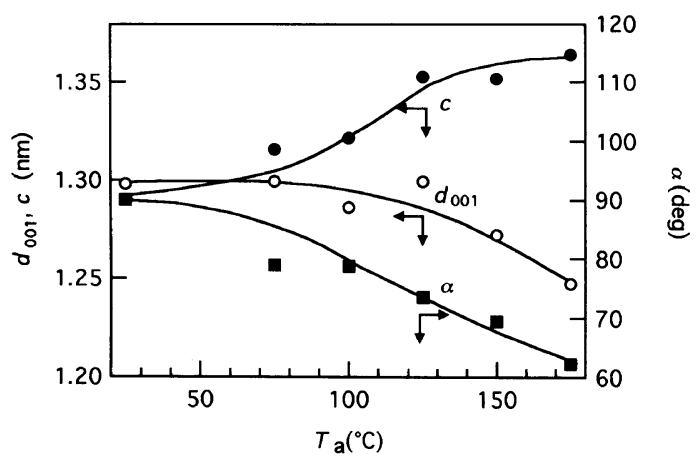

Figure 10. Annealing temperature dependence of $d_{001}, \alpha$, and $c$. crystals in the as-drawn and annealed sample as illustrated in Figure 11: The chains in the annealed sample have an all-trans conformation as shown in Figure 11 (b) while the chains in the as-drawn sample have a distorted conformation. One possible distorted conformation is the $\gamma$-form conformation ${ }^{10}$ as shown in Figure 11 (a). The amide group has a conformation of skew-trans-skew' and the fiber period $c$ is smaller than an all-trans conformation. This corresponds to the fact that the fiber period becomes larger in the annealed sample as shown in Figure 10. As the cross-sectional area is smaller in the annealed crystal, the packing is more compact. In addition, as the cross-section is more anisotropic, the steric hindrance during the rotation of a chain may be larger. Therefore the potential barrier against the chain rotation becomes larger in the annealed crystal to result in larger switching times as shown in Figure 3 and Figure 5.

In this model the as-drawn and the annealed crystal give nearly the same amount of dipole density and dipole moment. $d_{010}$ decreases by $9.5 \%$ while $c$ increase $5.5 \%$ giving an increase in dipole density of only several percent on annealing. Thus, this model is consistent with the experiment which gives little change in the amount of the reversed polarization on annealing.

It is to be noted that all amide groups in crystals are

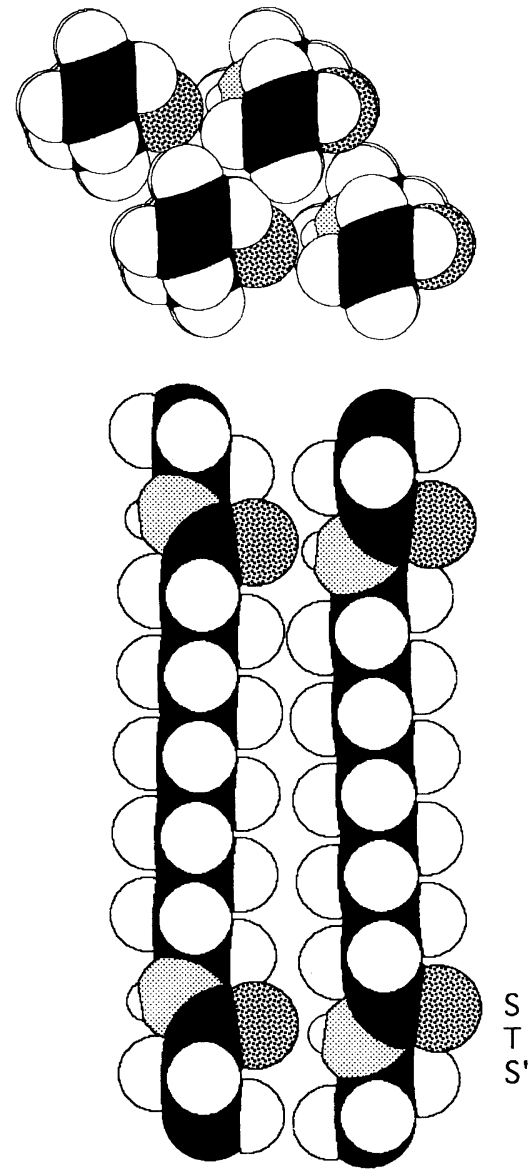

(a)
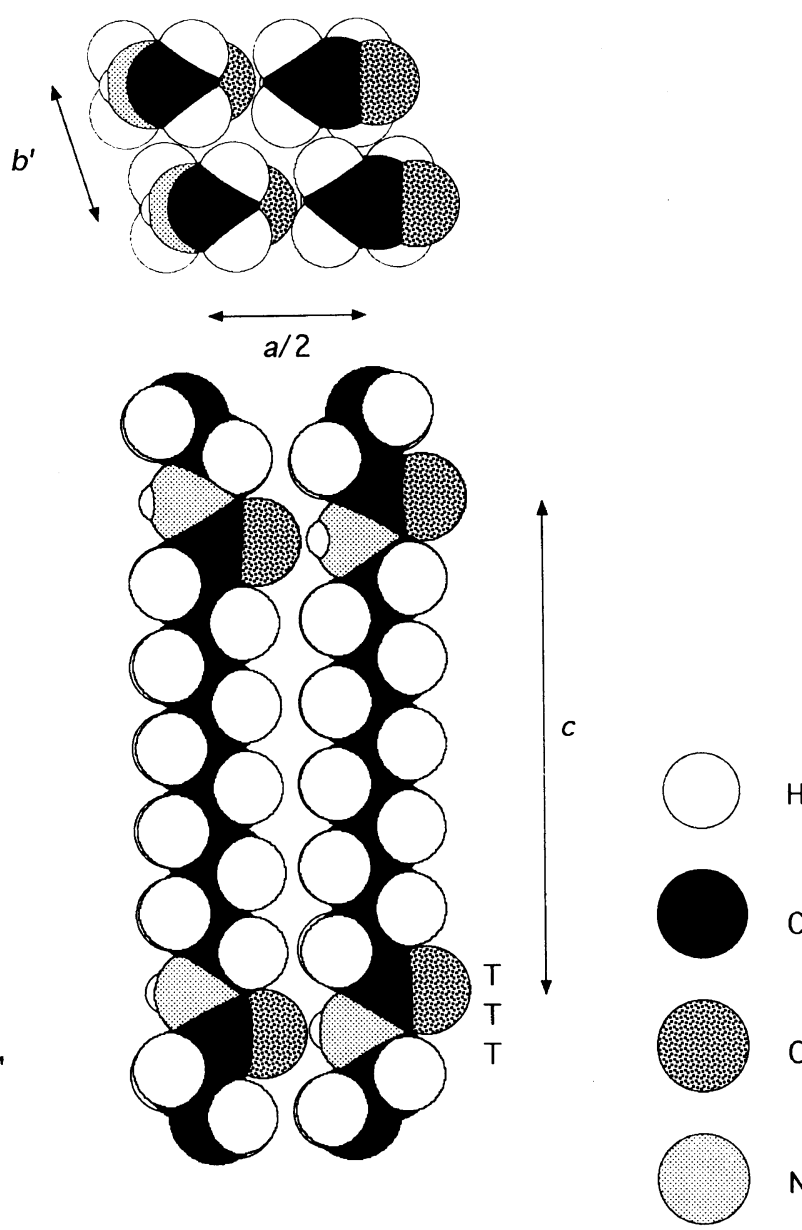

H

C $\mathrm{N}$

Figure 11. The model of the structural change from (a) as-drawn sample to (b) annealed sample. The cross-section of chains (upper) and the hydrogen-bonded sheet (lower) are shown. 
hydrogen-bonded to their neighbors in both crystal structures. Moreover, the distance between the hydrogenbonded chains, i.e., $a / 2$, does not change as shown in Figure 9: the strength of hydrogen-bonding is kept constant on annealing. Thus, the changes in dynamical properties are not caused by the strengthening of hydrogen-bonding in crystals.

As previously mentioned with regard to Figure 2, annealing is accompanied by broadening of switching curve. Several possible mechanism of this broadening can be pointed out: e.g., the broadening of the distribution of switching time due to inhomogenous structural change, an existence of some slowing mechanism in the polarization reversal, etc. However, the detail of the mechanism of broadening is not obvious.

We have shown that melt-quenched and uniaxially drawn Nylon 11 exhibits typical switching characteristics. Annealing at elevated temperatures causes much increase in the switching time but does not reduce the amount of reversed polarization. Structural investigation has shown that the crystal transformation occurs during annealing from a pseudohexagonal phase with distorted conformation to an all-trans triclinic phase. In both phase, the molecule dipoles associated with amide groups are align- ed in a parallel manner to produce the same amount of polarization. Shorter and anisotropic intermolecular distance in the latter generates larger potential barrier for rotation of molecules to yield slower switching process.

\section{REFERENCES}

1. T. Furukawa, Phase Transitions, 18, 143 (1989).

2. E. W. Jacobs and J. C. Hicks, Appl. Phys. Lett., 44, 402 (1984).

3. B. A. Newman, P. Chen, K. D. Pae, and J. I. Scheinbeim, J. Appl. Phys., 51, 5161 (1980).

4. S. C. Mathur, J. I. Scheinbeim, and B. A. Newman, J. Appl. Phys., 56, 2419 (1984).

5. J. W. Lee, Y. Takase, B. A. Newman, and J. I. Scheinbeim, J. Polym. Sci. B: Polym. Phys., 29, 273 (1991).

6. J. W. Lee, Y. Takase, B. A. Newman, and J. I. Scheinbeim, J. Polym. Sci. B: Polym. Phys., 29, 279 (1991).

7. S. Ikeda, T. Saito, M. Nonomura, and T. Koda, Ferroelectrics, 171, 329 (1995).

8. J. I. Scheinbeim, J. W. Lee, and B. A. Newman, Macromolecules, 25, 3729 (1992).

9. B. Z. Mei, J. I. Scheinbeim, and B. A. Newman, Ferroelectrics, 171, 177 (1995).

10. Y. Kinoshita, Makromol. Chem., 33, 1 (1959). 